Oelfke $\cdot$ Speditionsbetriebslehre in Frage und Antwort 
StD Dipl.-Hdl. Wolfgang Oelfke

\section{Speditionsbetriebslehre in Frage und Antwort}

13., gründlich überarbeitete und erweiterte Auflage

\section{GABLER}


CIP-Titelaufnahme der Deutschen Bibliothek

\section{Oelfke, Wolfgang:}

Speditionsbetriebslehre in Frage und Antwort / Wolfgang

Oelfke. - 13., gründlich überarbeitete und erweiterte

Auflage - Wiesbaden: Gabler, 1993 :

Oelfke, Wolfgang : Speditionskaufmann

1. Auflage 1963

2. Auflage 1967

3. Auflage 1971

4. Auflage 1973

5. Auflage 1976

6. Auflage 1978

7. Auflage 1980

8. Auflage 1984

9. Auflage 1984

10. Auflage 1985

11. Auflage 1988

12. Auflage 1990

13. Auflage 1993

Der Gabler Verlag ist ein Unternehmen der Verlagsgruppe Bertelsmann International.

(C) Betriebswirtschaftlicher Verlag Dr. Th. Gabler GmbH, Wiesbaden 1993

Lektorat: Brigitte Stolz-Dacol

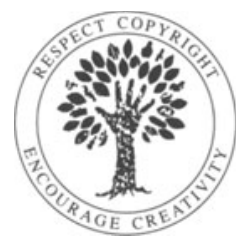

Das Werk einschließlich aller seiner Teile ist urheberrechtlich geschützt. Jede Verwertung außerhalb der engen Grenzen des Urheberrechtsgesetzes ist ohne Zustimmung des Verlags unzulässig und strafbar. Das gilt insbesondere für Vervielfältigungen, Übersetzungen, Mikroverfilmungen und die Einspeicherung und Verarbeitung in elektronischen Systemen.

Dieses Buch ist auf säurefreiem und chlorarm gebleichtem Papier gedruckt.

Die Wiedergabe von Gebrauchsnamen, Handelsnamen, Warenbezeichnungen usw. in diesem Werk berechtigt auch ohne besondere Kennzeichnung nicht zu der Annahme,daß solche Namen im Sinne der Warenzeichen- und Markenschutz-Gesetzgebung als frei zu betrachten wären und daher von jedermann benutzt werden dürften.

Satz: Satzstudio RESchulz, Dreieich-Buchschlag

ISBN-13: 978-3-409-97048-8 e-ISBN-13: 978-3-322-89240-9

DOI: $10.1007 / 978-3-322-89240-9$ 


\section{Vorwort zur 1. Auflage}

Die Aufgabe, die dem Güterverkehr in der Wirtschaft gestellt ist, liegt im Transport der Güter vom Ort der Erzeugung zum Ort des Verbrauches. Der Spediteur als Treuhänder seines Kunden und als neutraler Mittler besorgt diesen Gütertransport durch den Abschluß von Frachtverträgen mit Frachtführern und Verfrachtern; er führt auch den Gütertransport im Rahmen seines Selbsteintrittsrechtes als Frachtführer durch. Aus der Besorgung und der Selbstdurchführung des Gütertransportes ergibt sich eine Fülle von speditionellen Tätigkeiten, die zu ihrer Durchführung umfangreiche Kenntnisse erfordern. Besondere Anforderungen ergeben sich aus der Weltweite des Spediteurberufes und aus der außerordentlich starken Dynamik des Verkehrsgeschehens, die in der häufigen Änderung der zahllosen Verkehrsgesetze, -verordnungen und -tarife zum Ausdruck kommt. Das staatlich anerkannte Berufsbild des Speditionskaufmannes gibt einen Überblick über die Arbeitsgebiete des Spediteurs und über die Kenntnisse, die von einem kaufmännischen Angestellten in diesem vielseitigen Wirtschaftszweig verlangt werden.

Geistige Wendigkeit, die Bereitschaft, sich neuen wirtschaftlichen und rechtlichen Gegebenheiten anzupassen, die Fähigkeit zu sorgfältiger und schneller Arbeitsweise und eine gute Ausbildung müssen von dem in diesem Gewerbe stehenden kaufmännischen Angestellten verlangt werden, wenn er die ihm gestellten Aufgaben selbständig erfüllen will.

Dieses Buch wendet sich an alle, die im kaufmännischen Bereich des Verkehrswesens tätig sind. In erster Linie soll es die betriebliche Ausbildung des Speditionslehrlings unterstützen und ergänzen. Lernt der Lehrling im Betrieb die einzelnen Speditionszweige schrittweise kennen, so kann er sich aus dem Buch parallel zur betrieblichen Unterweisung und in Ergänzung zum Berufsschulunterricht die Antwort auf die Fragen holen, die sich aus dem jeweiligen Arbeitsgebiet ergeben. Absolviert er seine Lehre in einem stark spezialisierten Betrieb, kann er sich über die wichtigsten Fragen der Arbeitsgebiete informieren, die er in seinem Betrieb nicht kennenlernt. Ebenso kann es dem kaufmännischen Angestellten in allen Zweigen des Güterverkehrswesens eine brauchbare Hilfe zum Selbststudium sein, wenn er sein berufliches Wissen ergänzen oder wenn er sich über das Geschehen in verwandten Arbeitsbereichen unterrichten will. Für Lehrherren, Lehrlingsausbilder und Mitglieder der Prüfungsausschüsse der Industrie- und Handelskammern kann es als Hilfsmittel dienen, sich über den Leistungsstand der Lehrlinge zu informieren.

Allen denen, die die Arbeit an diesem Buch unterstützt haben, sei herzlich gedankt. 


\section{Vorwort zur 13., gründlich überarbeiteten und erweiterten Auflage}

Die „Verordnung über die Berufsausbildung zum Speditionskaufmann/zur Speditionskauffrau“, die am 1. August 1984 in Kraft trat, ordnet die betriebliche Ausbildung durch das Ausbildungsberufsbild und den Ausbildungsrahmenplan. Diese hier enthaltenen Fertigkeiten und Kenntnisse, die im Betrieb zu erwerben sind, sollen während des Berufsschulbesuches durch die Lerninhalte des Lehrplanes ergänzt werden.

Schwerpunkt der Ausbildung und damit auch der Abschlußprüfung vor der Industrieund Handelskammer liegt im Bereich „Speditionsbetriebslehre“ und „Praktische Übungen“ (mündliche Prüfung); sie machen zusammen mit „Wirtschafts- und Sozialkunde" fünf Sechstel der Abschlußprüfung aus. Die Fragen dieses Buches decken die wesentlichen Fertigkeiten und Kenntnisse sowie Lerninhalte, die der Prüfling in speditionspraxisbezogenen Aufgaben und Fällen nachweisen soll.

DiesesBuchin seinervorliegendenFassung entsprichtderAusbildungsverordnungund dem Bundesrahmenlehrplan. Seine Inhalte werden durch relativ kleine Auflagen kurzfristig derraschenEntwicklung in der Verkehrswirtschaft angepaßt. Dahereignetes sich zur Vorbereitung auf die Abschlußprüfung vor der Industrie- und Handelskammer.

Dieses Buch wird seit rund drei Jahrzehnten zur Wiederholung und Festigung des Grundwissens in der Speditionspraxis und in der kaufmännischen Berufsschule verwendet. Es ist eine wertvolle Ergänzung zu dem im Bundesgebiet eingeführten Schulbuch „Güterverkehr - Spedition - Logistik“ von Oelfke, das unter Mitarbeit von Brandenburg, Waschkau und D. Oelfke in der 29. Auflage im Verlag Dr. Max Gehlen, Bad Homburg v. d. H., erschienen ist.

Alle Abschnitte der vorliegenden Auflage sind gründlich überarbeitet und teilweise ergänzt worden. Eingearbeitet wurden die Änderungen der ADSP mit SVS/RVS und wesentliche Änderungen des Marktordnungssystems ab 1.1.1993 und 1994. Ein neuer Abschnitt über Grundbegriffe der Kosten- und Leistungsrechnung weist auf die erhöhte Bedeutung des betrieblichen Rechnungswesens hin, die sich als Folge des Tarifaufhebungsgesetzes zwangsläufig ergibt.

Der Auszubildende ist aufgefordert, den Stand und den Inhalt der Änderungen der Marktordnung in der Bundesrepublik und in der EG in Absprache mit seinem Ausbilder oder durch Selbststudium laufend zu überprüfen und sein Wissen auf den neuesten Stand der schnellen Entwicklung zu bringen.

Für jede Kritik bin ich den Nutzern meines Buches dankbar.

Hamburg, im April 1993

Wolfgang Oelfke 


\title{
Inhaltsverzeichnis
}

\author{
Vorwort
}

A. Aufgaben und rechtliche Stellung des Spediteurs in der Wirtschaft .... 1

I. Die rechtliche und wirtschaftliche Stellung des Spediteurs $\ldots \ldots \ldots \quad 1$

II. Die Stellung des Spediteurs zum Frachtführer, Verfrachter und Lagerhalter $\ldots \ldots \ldots \ldots \ldots \ldots \ldots \ldots \ldots \ldots \ldots \ldots \ldots \ldots \ldots, \quad 9$

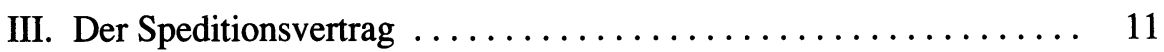

1. Die Vertragsgrundlagen nach dem Gesetz und nach den ADSp .. 11

2. Werbung und $A b s c h l u ß$ des Speditionsvertrages ........... 15

3. Die Erfüllung des Speditionsvertrages $\ldots \ldots \ldots \ldots \ldots \ldots \ldots .20$

4. Das Pfand- und Zurückbehaltungsrecht $\ldots \ldots \ldots \ldots \ldots \ldots \ldots .23$

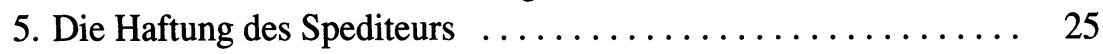

6. Die Speditionsversicherung $\ldots \ldots \ldots \ldots \ldots \ldots \ldots \ldots \ldots .29$

IV. Die Grundlagen des Frachtvertrages nach dem HGB $\ldots \ldots \ldots \ldots$

B. Das Speditionsunternehmen $\ldots \ldots \ldots \ldots \ldots \ldots \ldots \ldots \ldots \ldots \ldots$

I. Der Mensch im Betrieb ........................ 39

1. Der Kaufmann . ............................ 39

2. Die Mitarbeiter und Mitarbeiterinnen des Kaufmanns ....... 41

3. Die Vollmachten $\ldots \ldots \ldots \ldots \ldots \ldots \ldots \ldots \ldots \ldots \ldots, 45$

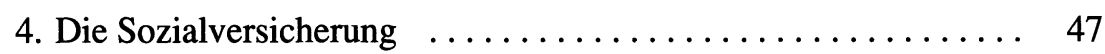

5. Organisation und Rechtsschutz der Arbeitnehmer ......... 50

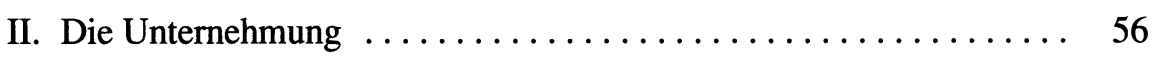

1. Gründung, Handelsregister und Firma $\ldots \ldots \ldots \ldots \ldots \ldots \ldots .56$

2. Die Unternehmungsformen $\ldots \ldots \ldots \ldots \ldots \ldots \ldots \ldots \ldots \ldots$

3. Die Unternehmungszusammenschlüsse $\ldots \ldots \ldots \ldots \ldots \ldots \ldots .65$

4. Die Unternehmung in Zahlungsschwierigkeiten $\ldots \ldots \ldots \ldots \ldots 67$

III. Der allgemeine kaufmännische Geschäftsverkehr $\ldots \ldots \ldots \ldots \ldots .69$

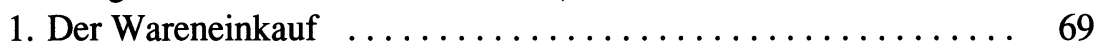

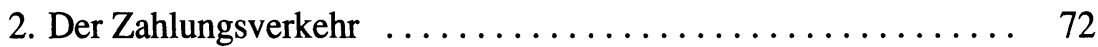

3. Der Einzug von Forderungen $\ldots \ldots \ldots \ldots \ldots \ldots \ldots \ldots \ldots \ldots$

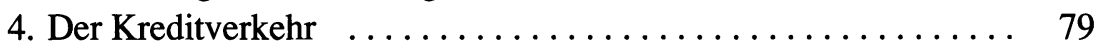


C. Die einzelnen Speditionszweige $\ldots \ldots \ldots \ldots \ldots \ldots \ldots \ldots \ldots \ldots \ldots \ldots \ldots$

I. Die Lkw-Spedition $\ldots \ldots \ldots \ldots \ldots \ldots \ldots \ldots \ldots \ldots \ldots \ldots \ldots \ldots$

1. Der Güterverkehr mit dem Lkw ................. 84

2. Der internationale Straßengüterverkehr ............. 108

3. Der Spediteur im Lkw-Verkehr . ................ 113

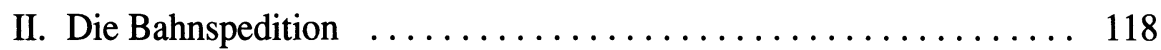

1. Die Deutsche Bundesbahn als Frachtführer ............ 118

2. Der internationale Eisenbahngüterverkehr ............. 134

3. Der Spediteur als Vertragspartner der Deutschen Bundesbahn .. 136

III. Der Spediteursammelgutverkehr $\ldots \ldots \ldots \ldots \ldots \ldots \ldots \ldots . \ldots \ldots$

IV. Außenwirtschaftliche und zollrechtliche Grundkenntnisse für den internationalen Spediteur $\ldots \ldots \ldots \ldots \ldots \ldots \ldots . \ldots \ldots$

1. Die Außenwirtschaft ........................ 144

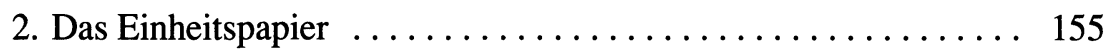

3. Das Zollwesen $\ldots \ldots \ldots \ldots \ldots \ldots \ldots \ldots \ldots \ldots \ldots \ldots \ldots \ldots$

V. Die Seehafenspedition $\ldots \ldots \ldots \ldots \ldots \ldots \ldots \ldots \ldots \ldots \ldots$

1. Der Güterverkehr mit dem Seeschiff $\ldots \ldots \ldots \ldots \ldots \ldots \ldots$

2. Die Exportspedition ........................ 178

3. Die Importspedition $\ldots \ldots \ldots \ldots \ldots \ldots \ldots \ldots \ldots \ldots \ldots \ldots$

VI. Die Luftfrachtspedition ....................... 191

VII. Die Binnenschiffahrtsspedition $\ldots \ldots \ldots \ldots \ldots \ldots \ldots \ldots \ldots .201$

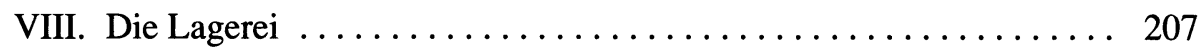

IX. Über die Preisbildung in der Spedition und im Güterverkehr $\ldots \ldots 215$

D. Zur politischen Ordnung in der Bundesrepublik Deutschland ...... 224

I. Das Grundgesetz und die Grundrechte $\ldots \ldots \ldots \ldots \ldots \ldots \ldots .224$

II. Die Bundesrepublik Deutschland als demokratischer
und sozialer Rechtsstaat $\ldots \ldots \ldots \ldots \ldots \ldots \ldots \ldots \ldots \ldots \ldots \ldots \ldots$

III. Die Parteien $\ldots \ldots \ldots \ldots \ldots \ldots \ldots \ldots \ldots \ldots \ldots \ldots . \ldots \ldots$

IV. Die Bundestagswahlen ....................... 231

V. Die Verfassungsorgane und ihre Arbeit $\ldots \ldots \ldots \ldots \ldots \ldots \ldots .233$

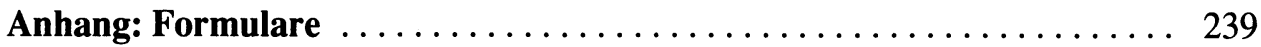

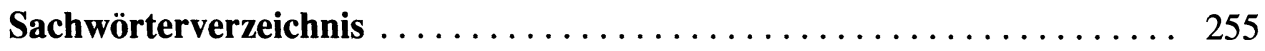

